\title{
Weniger Schwangerschafts-Komplikationen mit kontinuierlicher Glukosemessung
}

\begin{abstract}
Schwangere mit Typ-1-Diabetes profitieren von einer kontinuierlichen Glukosemessung. Nicht nur die Blutzuckerkontrolle der Mutter ist besser als mit der gängigen Blutzuckerselbstmessung. Es kam damit auch zu weniger Komplikationen beim Neugeborenen.
\end{abstract}

Schwangere mit Typ-1-Diabetes sollten ihre Blutzuckerspiegel wohl möglichst mithilfe einer kontinuierlichen Glukosemessung (CGM) bestimmen. Mit dieser Messmethode traten in der randomisierten CONCEPTT-Studie weniger Schwangerschaftskomplikationen auf als mit der üblichen Blutzuckerselbstmessung. Die Autorinnen um Denice Feig vom Mt Sinai Hospital sprechen sich deshalb dafür aus, allen Schwangeren mit Typ1-Diabetes innerhalb des ersten Schwangerschafts-Trimesters eine kontinuierliche Glukosemessung anzubieten. „Es müssen gerademal sechs Frauen diese Methode anwenden, damit ein Kind weniger mit einer Makrosomie zu Welt kommt", berichten Feig und ihre Kollegin Helen Murphy von der University of East Anglia auf einer Pressekonferenz des EASD-Kongresses.

\section{Weniger Neugeborene mit Makrosomie}

Noch immer sind die Komplikationsraten bei Schwangeren mit Diabetes recht noch, u. a. deshalb, weil selbst motivierte Frauen häufig keine optimale Blutzuckerkontrolle erreichen. So ist das Risiko, dass das Neugeborene mit einem zu hohen Geburtsgewicht auf die Welt kommt, fünfmal höher als bei gesunden Frauen. Studien zufolge kann die Anwendung einer kontinuierlichen Glukosemessung die Blutzuckerkontrolle verbessern. Und wie die Ergebnisse der CONCEPTT-Studie mit 215 Frauen nun belegen, führt dies letztlich auch zu einer Reduktion der Komplikationsraten bei Schwangeren mit Typ-1-Diabetes. So gebaren fast zwei Drittel aller Studienteilnehmerinnen in der Kontroll- gruppe (69\%) ein zu schweres Kind. In der Gruppe mit CGM waren dagegen nur $53 \%$ der Kinder betroffen.

Die Neugeborenen der Mütter, die die CGM nutzten, benötigten seltener eine intravenöse Glukose-Injektion aufgrund gefährlich niedriger Glukosewerte als die Kinder in der Kontrollgruppe (15 vs. $28 \%$ ). Sie verbrachten auch weniger Zeit auf einer Neugeborenen-Intensivstation (27 vs. $43 \%$ waren länger als 24 Stunden dort). Generell war die Krankenhausverweildauer für Frauen, die die CGM anwendeten, ein Tag kürzer.

\section{Bessere Blutzuckerkontrolle}

Das $\mathrm{HbA}_{1 \mathrm{c}}$ verbesserte sich bis zur 34. Schwangerschaftswoche bei allen Frauen, bei den CGM-Nutzerinnen war die Veränderung des $\mathrm{HbA}_{1 c}$ - der primäre Endpunkt - jedoch noch deutlicher $(-0,19 \%)$. Auch lagen ihre Blutzuckerwerte häufiger im Zielbereich von $3,5-7,8 \mathrm{mmol} / \mathrm{L}$ (68 vs. $61 \%$ der Zeit) und im Schnitt 72 Minuten pro Tag seltener in einem hyperglykämischen Bereich ( $>7,8 \mathrm{mmol}$ ) als die Werte in der Kontrollgruppe.

In dieser zweiten Phase der multizentrischen CONCEPTTStudie wurden 215 Schwangere mit Typ-1-Diabetes und suboptimaler Glukosekontrolle $\left(\mathrm{HbA}_{1 \mathrm{c}} \geq 6,5 \%\right)$ ab dem ersten Trimester randomisiert entweder zur CGM-Gruppe oder zur üblichen Blutzuckerselbstmessung zugeteilt. Alle Frauen trugen eine Insulinpumpe oder spritzten sich Insulin. Das CGM-Device war in der Lage, 288 Glukosewerte pro Tag aufzuzeichnen. Mit der traditionellen Methode bestimmten die Frauen vier- bis achtmal pro Tag ihre Glukosespiegel.

Veronika Schlimpert

Referenzen: Feig D et al. Continuous glucose monitoring in pregnant women with type 1 diabetes (CONCEPTT): a multicentre international randomised controlled trial. The Lancet 2017; http://dx.doi.org/10.1016/S0140-6736(17)32400Pressekonferenz: Continuous Glucose Monitoring in Women with Typ 1 Diabetes in Pregnancy Trial, EASD Annual Meeting, 14.09.2017 in Lissabon

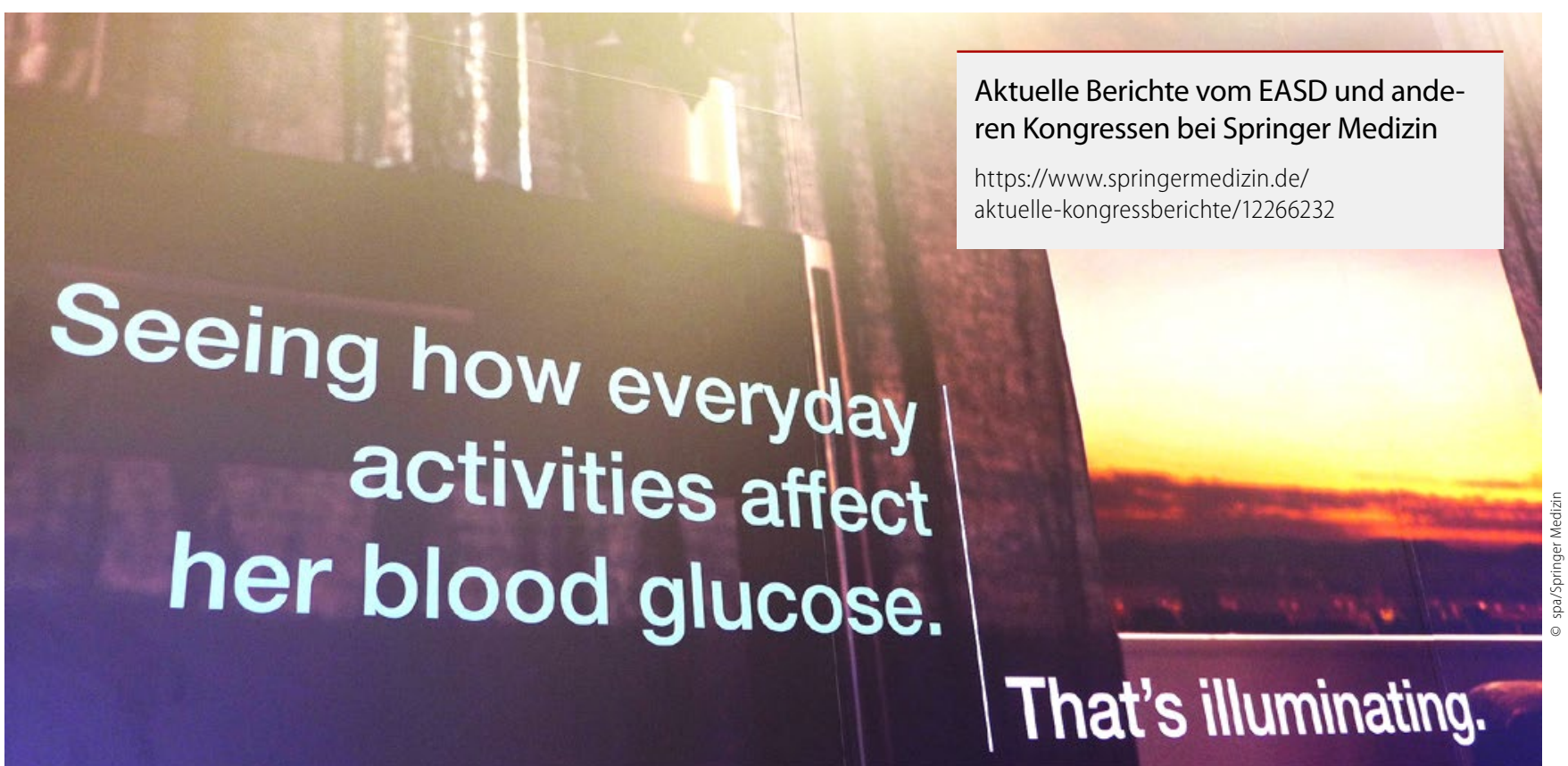

